\title{
The virtual element method in 50 lines of MATLAB
}

\author{
Oliver J. Sutton ${ }^{1}$ (iD
}

Received: 20 April 2016 / Accepted: 10 November 2016/ Published online: 3 December 2016 (C) The Author(s) 2016. This article is published with open access at Springerlink.com

\begin{abstract}
We present a 50-line MATLAB implementation of the lowest order virtual element method for the two-dimensional Poisson problem on general polygonal meshes. The matrix formulation of the method is discussed, along with the structure of the overall algorithm for computing with a virtual element method. The purpose of this software is primarily educational, to demonstrate how the key components of the method can be translated into code.
\end{abstract}

Keywords Virtual element method · Polygonal meshes · MATLAB implementation

\section{Introduction}

The virtual element method, introduced in [6], is a generalisation of the standard conforming finite element method for the approximation of solutions to partial differential equations. The method is designed in such a way as to enable the construction of high order approximation spaces which may include an arbitrary degree of global regularity [10] on meshes consisting of very general polygonal (or polyhedral) elements. This cocktail of desirable features has attracted the method a lot of attention (see, for example, [1, 3, 4, 11, 12, 14, 21, 30]) and is made possible through the virtual element space of trial and test functions, which is implicitly defined on each mesh element through local PDE problems. These local problems are designed in such a way that the virtual element space includes a

Oliver J. Sutton

ojs4@le.ac.uk

1 Department of Mathematics, University of Leicester, University Road, Leicester, LE1 7RH, UK 
subspace of polynomials of some prescribed degree (referred to as the degree of the method) alongside other, typically unknown, virtual functions. In this respect, and like many other conforming approaches to polygonal meshes such as the polygonal finite element method [18, 27] or BEM-based FEM [22], the virtual element method falls within the broad class of generalised finite element methods [25]. What sets the virtual element method apart from these other approaches, however, is that the extra non-polynomial virtual functions never need to be determined or evaluated in practice. Instead, they are understood and used solely through certain defining properties of the virtual element space and through their degrees of freedom, which, along with the discrete bilinear form, are carefully selected to ensure that the final stiffness matrix can be directly and exactly computed.

There is a history of short, simple codes being used to demonstrate the practical implementation details of various aspects of finite element methods. We refer, for instance, to the 'Remarks around 50 lines of MATLAB' paper [2] which presented a simple and transparent MATLAB implementation of the conforming finite element method, the 99-line topology optimisation code presented in [23], or the mixedFEM implementations presented by [5]. The purpose of these codes is primarily to demonstrate how the theoretical concepts can be distilled into code, although they typically fall at the opposite end of the educational spectrum from many other educational finite element libraries such as FreeFEM++ [16], FEniCS [17], or FEAP [29], amongst many others, or commercially available 'black-box' finite element software packages. Such software packages are typically designed to hide many of the actual implementation details of the methods they implement behind an intuitive interface which may automatically generate the necessary code directly from the weak form of the problem or simply a description of the physical system. Instead, the focus of these minimalist codes is on elucidating the 'behind-the-scenes' work necessary to construct and solve the matrix form of the method in a specific case. This is a tradition which we continue here, presenting a 50-line MATLAB implementation of the lowest order virtual element method for the Poisson problem in $2 \mathrm{D}$ on general polygonal meshes.

To the best of our knowledge, this is the first publicly available implementation of the virtual element method, although there are various references which discuss the matrix formulation of the method, see for instance [7, 14, 15]. In particular, [7] contains detailed explanations of the formulation of the terms in the matrix equations for the high order virtual element method applied to a reaction-diffusion problem. In many ways, the work here can be seen as a spiritual successor to [7] in the sense that while we restrict ourselves to just the linear virtual element method for the Poisson problem, we take the process one step further and provide a clear, useable MATLAB implementation of the method. The full code of the implementation is available from Netlib (http://www.netlib.org/numeralgo/) as the na45 package, and includes several examples of polygonal meshes.

The remainder of this work is structured as follows. In Section 2, we present the model problem of the Poisson problem. A very brief introduction to the virtual element framework is presented in Section 3, with a discussion of the discrete function spaces and bilinear forms. The details of the implementation of this method are given in Section 4, where we derive the matrix form of the discrete problem and show how 
this may be computed in practice. Section 5 contains a brief explanation of how to run the code using MATLAB. Finally, we offer some concluding remarks and ideas for possible extensions to the code in Section 6.

\section{Model problem}

Let $\Omega \subset \mathfrak{R}^{2}$ be a polygonal domain and consider the Poisson problem

$$
\begin{aligned}
-\Delta u=f & \text { in } \Omega, \\
u=g & \text { on } \partial \Omega,
\end{aligned}
$$

with $f \in L^{2}(\Omega)$ and $g \in H^{1 / 2}(\partial \Omega)$. This problem can be written in variational form as: find $u \in H_{g}^{1}(\Omega):=\left\{w \in H^{1}(\Omega): w=g\right.$ on $\left.\partial \Omega\right\}$ such that

$$
a(u, v):=(\nabla u, \nabla v)=(f, v)=: \ell(v) \quad \forall v \in H_{0}^{1}(\Omega)
$$

where $(\cdot, \cdot)$ denotes the standard $L^{2}(\Omega)$ inner product. This variational problem possesses a unique solution by the Lax-Milgram lemma.

\section{The virtual element method}

Let $\mathcal{T}_{h}$ be a family of partitions of the domain $\Omega$ into non-overlapping polygonal elements with maximum diameter $h$ whose boundaries are not self-intersecting. A theoretical analysis of the method, as given in [6], requires certain additional assumptions on the regularity of the mesh. Although we do not specifically state the assumptions here, the resulting set of requirements is general enough to include polygonal elements consisting of an arbitrary (but uniformly bounded) number of edges, which may also be non-convex. Moreover, recent work in [9] suggests that these mesh requirements may be relaxed even further to include meshes with arbitrarily small edges. To simplify the implementation, we restrict the mesh to include only elements which contain their own centroid, as defined in (4.4). We note that this class of elements includes those with co-planar edges, as commonly found in locally refined meshes with hanging nodes, and even non-convex elements.

The vertices of a polygonal element $E$ with $N^{E}$ edges are denoted by $v_{i}$ for $i=$ $1, \ldots, N^{E}$, and we adopt the convention that the edge $e_{i}$ connects $v_{i}$ and $v_{i+1}$, where the indices are understood to wrap within the range 1 to $N^{E}$.

\subsection{Virtual element function spaces}

The discrete function space is defined to be

$$
V_{h}:=\left\{v_{h} \in H_{g}^{1}(\Omega):\left.v_{h}\right|_{E} \in V_{h}^{E} \text { for all } E \in \mathcal{T}_{h}\right\}
$$

where the local space $V_{h}^{E}$ on the element $E$ can be understood through the following three properties: 
- $V_{h}^{E}$ includes the space $\mathcal{P}_{E}$ of physical-frame polynomials on $E$ with total degree $\leq 1$ as a subspace.

- Any function in $V_{h}^{E}$ can be uniquely identified by its values at the vertices of $E$, which are taken to be the degrees of freedom of the space. We note that this implies that the dimension of the space $V_{h}^{E}$ is equal to $N^{E}$.

- Every function in $V_{h}^{E}$ is a linear polynomial on each edge of $E$.

The subspace of linear polynomials provides the approximation power of the virtual element space, and is responsible for the accuracy of the method. On triangular elements, the space consists entirely of these linear polynomials, and thus, the method reduces to the standard linear finite element method. However, on more general shaped polygonal elements, the space will also include other, implicitly defined, 'virtual' functions, cf. (3.3). The method is designed in such a way that these will never need to be explicitly computed or evaluated and are instead understood solely through their values at the vertices of $E$, which we take to be the degrees of freedom of the space $V_{h}^{E}$. In this respect, the virtual element space can be seen as a straightforward generalisation of the standard linear conforming finite element space on triangles to more general shaped elements.

The first observation we make about this space is that just the properties outlined above allow us to compute the Ritz projection $\Pi^{E}: V_{h}^{E} \rightarrow \mathcal{P}_{E}$ of any function in the local virtual element space $V_{h}^{E}$ onto the subspace of linear polynomials. This projection is defined for $v_{h} \in V_{h}^{E}$ by the conditions

$$
\left\{\begin{array}{l}
\frac{\left(\nabla\left(\Pi^{E} v_{h}-v_{h}\right), \nabla p\right)_{0, E}=0 \text { for all } p \in \mathcal{P}_{E},}{\Pi^{E} v_{h}}=\overline{v_{h}},
\end{array}\right.
$$

where $\overline{w_{h}}:=\frac{1}{N^{E}} \sum_{i=1}^{N^{E}} w_{h}\left(v_{i}\right)$ denotes the average value of $w_{h}$ at the vertices of $E$. This second condition is necessary to fix the constant part of $\Pi^{E} v_{h}$ and is clearly computable for any $v_{h} \in V_{h}^{E}$ from just its degrees of freedom.

From (3.1), the divergence theorem, and the fact that the Laplacian of a linear function is zero, we have that, for any $v_{h} \in V_{h}^{E}$ and $p \in \mathcal{P}_{E}$,

$$
\left(\nabla \Pi^{E} v_{h}, \nabla p\right)_{0, E}=\left(\nabla v_{h}, \nabla p\right)_{0, E}=\sum_{e \in \partial E} \int_{e} v_{h} n_{e} \cdot \nabla p \mathrm{~d} s,
$$

where $n_{e}$ denotes the unit normal vector to the edge $e$ directed out of the element $E$. The final expression on the right hand side here can be exactly evaluated since $v_{h}$ is a linear polynomial on each edge of $E$, entirely determined by its values at the vertices, while the gradient of the linear polynomial $p$ is a known constant. By picking a basis for the polynomial space $\mathcal{P}_{E}$, (3.2) can be written as a matrix problem which can be solved to find the coefficients of $\Pi^{E} v_{h}$ with respect to this polynomial basis. We will come back to this in Section 4, although for now we just rely on the fact that this projection is computable. 
The actual definition of the virtual element space which we use here is the lowest order space introduced in [6], given by

$$
\begin{aligned}
V_{h}^{E}:=\left\{v \in H^{1}(E):\right. & \Delta v=0,\left.\quad v\right|_{\partial E} \in C^{0}(\partial E), \\
& \left.\left.v\right|_{e} \in \mathcal{P}_{e} \text { for each } e \in \partial E\right\},
\end{aligned}
$$

where $\mathcal{P}_{e}$ denotes the space of linear polynomials on the edge $e$. The fact that the vertex values can be used as degrees of freedom to describe this space is proven in [6].

The global degrees of freedom for $V_{h}$ are simply taken to be the value of the function at each vertex in the mesh, thus imposing the continuity of the ambient space. The degrees of freedom at vertices on the domain boundary are fixed in accordance with the boundary condition. The dimension of the global virtual element space $V_{h}$ shall be denoted by $N$.

\subsection{Discrete bilinear form}

Define the bilinear form $a^{E}: H^{1}(E) \times H^{1}(E) \rightarrow \Re$ to be the restriction of $a$ to the element $E$, i.e. $a^{E}(v, w):=(\nabla v, \nabla w)_{0, E}$ for any $v, w \in H^{1}(E)$. Following [6], we introduce the discrete counterpart $a_{h}^{E}: V_{h}^{E} \times V_{h}^{E} \rightarrow \Re$ of $a^{E}$ which we define as

$$
a_{h}^{E}\left(v_{h}, w_{h}\right):=\left(\nabla \Pi^{E} v_{h}, \nabla \Pi^{E} w_{h}\right)_{0, E}+S^{E}\left(v_{h}-\Pi^{E} v_{h}, w_{h}-\Pi^{E} w_{h}\right),
$$

with

$$
S^{E}\left(v_{h}, w_{h}\right):=\sum_{r=1}^{N^{E}} \operatorname{dof}_{r}\left(v_{h}\right) \operatorname{dof}_{r}\left(w_{h}\right),
$$

where $\operatorname{dof}_{r}\left(v_{h}\right)$ denotes the value of the $r$ th local degree of freedom defining $v_{h}$ in $V_{h}^{E}$ with respect to some arbitrary (but fixed) ordering ${ }^{1}$. This means that $S^{E}$ is simply the Euclidean inner product between vectors of degrees of freedom. Finally, we define

$$
a_{h}\left(v_{h}, w_{h}\right):=\sum_{E \in \mathcal{T}_{h}} a_{h}^{E}\left(v_{h}, w_{h}\right),
$$

to be the discrete counterpart of $a$.

Crucial to the method is the observation that the local discrete bilinear forms satisfy the following two properties [6]:

- Polynomial consistency: for any $v_{h} \in V_{h}^{E}$ and $p \in \mathcal{P}_{E}$,

$$
a_{h}^{E}\left(v_{h}, p\right)=a^{E}\left(v_{h}, p\right) .
$$

- Stability: there exists a constant $C_{\text {stab }}$ independent of $h$ and $E$ such that

$$
C_{s t a b}^{-1} a^{E}\left(v_{h}, v_{h}\right) \leq a_{h}^{E}\left(v_{h}, v_{h}\right) \leq C_{s t a b} a^{E}\left(v_{h}, v_{h}\right),
$$

for any $v_{h} \in V_{h}^{E}$.

The requirement of polynomial consistency implies that the method satisfies the patch test commonly used in the engineering literature, expressing the fact that the method

\footnotetext{
${ }^{1}$ For instance, this could be achieved simply by numbering the vertices of the polygon $E$.
} 
is exact when the solution is a piecewise linear polynomial with respect to the mesh $\mathcal{T}_{h}$ and provides the accuracy of the method. The stability property, on the other hand, ensures that the discrete bilinear form inherits the continuity and coercivity of the original variational form $a$, as proven in [6]. In the final matrix formulation of the problem, this property can be viewed as ensuring that the problem stiffness matrix is of the correct rank.

In light of these two properties, the two terms of $a_{h}^{E}$ are referred to as the consistency and stabilising terms respectively since only the first term is non-zero when either $v_{h}$ or $w_{h}$ is a polynomial, and thus single-handedly ensures that the polynomial consistency property is satisfied, while the second term ensures that the stability property is satisfied even when $v_{h}$ or $w_{h}$ are in the kernel of $\Pi^{E}$. For a proof that this choice of stabilising term $S^{E}$ does indeed satisfy the stability property, we refer to $[9,14]$.

Moreover, both terms of $a_{h}^{E}$ in (3.4) are computable using just the degrees of freedom of the virtual element space (to compute the projector $\Pi^{E}$ and to evaluate the stabilising term) and knowledge of the polynomial subspace $\mathcal{P}_{E}$ (to evaluate the consistency term of $a_{h}^{E}$, which is made of integrals of polynomials, just like in a standard finite element method). This will be further demonstrated in Section 4, where it will also become apparent that this particular virtual element method can be implemented without requiring any quadrature to compute the stiffness matrix, although a very simple barycentric quadrature scheme is used to evaluate the forcing term.

Still following [6], the linear form $\ell$ on the right-hand side of the variational problem (2.2) is discretised by $\ell_{h}: V_{h}^{E} \rightarrow \Re$ such that

$$
\ell_{h}\left(v_{h}\right):=\sum_{E \in T_{h}}\left(\Pi_{0}^{E} f, \overline{v_{h}}\right)_{0, E},
$$

where $\Pi_{0}^{E}: V_{h}^{E} \rightarrow \Re$ denotes the $L^{2}(E)$-orthogonal projection onto constants, defined for any $w_{h} \in V_{h}^{E}$ to be such that

$$
\int_{E}\left(w_{h}-\Pi_{0}^{E} w_{h}\right) d x=0 .
$$

The discrete problem which we solve can then be written as: find $u_{h} \in V_{h}$ such that

$$
a_{h}\left(u_{h}, v_{h}\right)=\ell_{h}\left(v_{h}\right),
$$

for all $v_{h} \in V_{h}^{E}$.

\section{Implementation}

As with a typical finite element method, we start by introducing the Lagrangian basis $\left\{\varphi_{i}\right\}_{i=1}^{N}$ of $V_{h}$ with respect to the global set of degrees of freedom, defined by the property that $\varphi_{i}\left(v_{j}\right)=\delta_{i j}$, where $\delta_{i j}$ is the Kronecker delta. We also introduce the Lagrangian basis of the local virtual element space $V_{h}^{E}$ on the element $E$ as $\left\{\varphi_{i}^{E}\right\}_{i=1}^{N_{E}}$, defined by the local equivalent of the same property. 
We will also need a basis for the space $\mathcal{P}_{E}$ of local physical frame linear polynomials on each element $E$. Many choices are possible here, although in keeping with the convention commonly adopted in the literature on virtual element methods, we choose the set of scaled monomials of degree 1 . These are defined on the element $E$ as

$$
\mathcal{M}_{E}:=\left\{m_{1}(x, y):=1, \quad m_{2}(x, y):=\frac{x-x_{E}}{h_{E}}, \quad m_{3}(x, y):=\frac{y-y_{E}}{h_{E}}\right\},
$$

where $x_{E}$ and $y_{E}$ respectively denote the $x$ and $y$ coordinates of the centroid of the element in the standard Cartesian coordinate system, and $h_{E}$ is the diameter of the element $E$. We denote by $N_{\mathcal{P}}=3$ the cardinality of this basis and therefore the dimension of $\mathcal{P}_{E}$

In the hope of avoiding confusion, we adopt the convention of indexing coefficients and basis functions in the basis of $V_{h}^{E}$ using Latin letters, while those of $\mathcal{P}_{E}$ will be indexed using Greek letters.

With these two bases at our disposal, we can now derive the matrix form of the discrete problem (3.6). Expanding the virtual element solution $u_{h}$ as

$$
u_{h}=\sum_{i=1}^{N} U_{i} \varphi_{i}
$$

problem (3.6) can be rewritten using the definitions (3.4) of $a_{h}^{E}$ and (3.5) of $\ell_{h}$ as: find $U \in \mathfrak{R}^{N}$ such that

$$
\sum_{i=1}^{N} U_{i} \sum_{E \in \mathcal{T}_{h}}\left(\left(\nabla \Pi^{E} \varphi_{i}, \nabla \Pi^{E} \varphi_{j}\right)_{0, E}+S^{E}\left(\varphi_{i}-\Pi^{E} \varphi_{i}, \varphi_{j}-\Pi^{E} \varphi_{j}\right)\right)=\sum_{E}\left(\Pi_{0}^{E} f, \overline{\varphi_{j}}\right)_{0, E}
$$

for $j=1, \ldots, N$. This may be expressed in matrix form as $K U=F$ where

$$
K_{j, i}=\sum_{E \in \mathcal{T}_{h}}\left(\left(\nabla \Pi^{E} \varphi_{i}, \nabla \Pi^{E} \varphi_{j}\right)_{0, E}+S^{E}\left(\varphi_{i}-\Pi^{E} \varphi_{i}, \varphi_{j}-\Pi^{E} \varphi_{j}\right)\right), \quad F_{j}=\sum_{E}\left(\Pi_{0}^{E} f, \overline{\varphi_{j}}\right)_{0, E},
$$

for $i, j=1, \ldots, N$. Since both these terms are defined through sums over elements, the obvious way to compute the entries of $K$ and $F$ is by computing the non-zero local contributions from each element $E$ in the form of the local stiffness matrix $K^{E} \in \mathfrak{R}^{N^{E} \times N^{E}}$ and local forcing vector $F^{E} \in \mathbb{R}^{N^{E}}$, given by

$K_{j, i}^{E}=\left(\nabla \Pi^{E} \varphi_{i}^{E}, \nabla \Pi^{E} \varphi_{j}^{E}\right)_{0, E}+S^{E}\left(\varphi_{i}^{E}-\Pi^{E} \varphi_{i}^{E}, \varphi_{j}^{E}-\Pi^{E} \varphi_{j}^{E}\right), \quad F_{j}^{E}:=\left(\Pi_{0}^{E} f, \overline{\varphi_{j}^{E}}\right)_{0, E}$,

for $i, j=1, \ldots, N^{E}$, and adding them into the corresponding entries of $K$ and $F$.

This, of course, dictates that the overall structure of a virtual element method implementation will be much the same as for a standard finite element method, as outlined in Algorithm 1. The key point of departure from the standard finite element method is in how the element stiffness matrices should be calculated. Firstly, the computation of the local stiffness matrices relies on first computing the local 
Ritz projector $\Pi^{E}$ on each element. Secondly, where the implementation of a conventional finite element might rely on a mapping to a reference element, no such equivalent process is possible here because the mesh elements are allowed to be general (possibly non-convex) polygons.

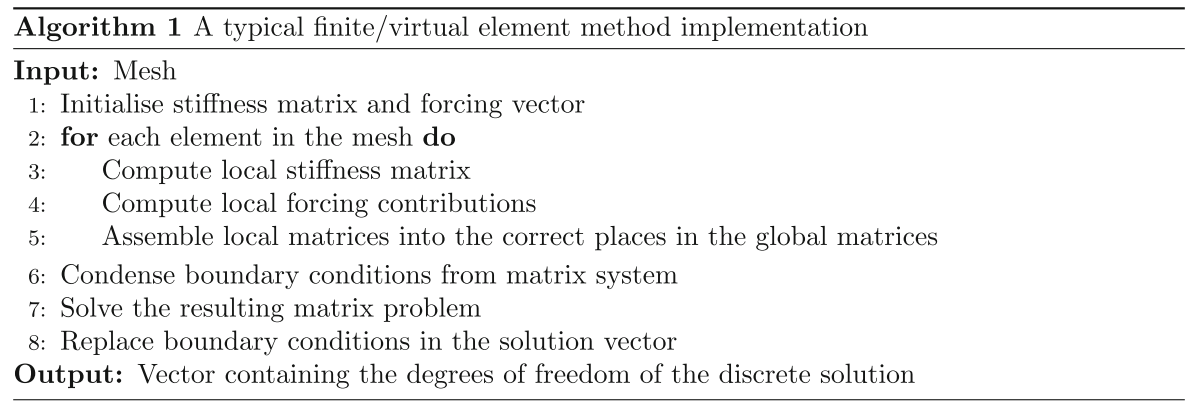

The main code of the implementation is shown in Listing 1. In the remainder of this section, we dissect the code to highlight how the various steps outlined in Algorithm 1 can be implemented in code. Much of the matrix formulation presented in this section is similar to that in [7], although here we focus specifically on the case of the lowest order method and take the process a step further to include the details of how each step is accomplished in the code. The full code also relies on several auxiliary functions:

- square_domain_rhs.m: contains the definition of the forcing function

$$
f(x, y)=15 \sin (\pi x) \sin (\pi y),
$$

- square_domain_boundary_condition.m: contains the definition of the Dirichlet boundary condition

$$
g(x, y)=x y \sin (\pi x),
$$

- L_domain_rhs.m: contains the definition of the forcing function

$$
f(x, y)=0,
$$

- L_domain_boundary_condition.m: contains the definition of the Dirichlet boundary condition

$$
g(r, \theta)=r^{2 / 3} \sin \left(\frac{2 \theta-\pi}{3}\right),
$$

where $r$ and $\theta$ are the standard polar coordinates centred at the origin

- plot_solution.m: produces a MATLAB figure containing a plot of the approximate solution $u_{h}$. Note that this plot is generated using the values of $u_{h}$ at the vertices of the mesh, which are interpolated by the MATLAB patch function to produce a surface.

The implementations of these functions are available as the package na45 in the NUMERALGO library from Netlib, along with the vem.m file shown in Listing 1 


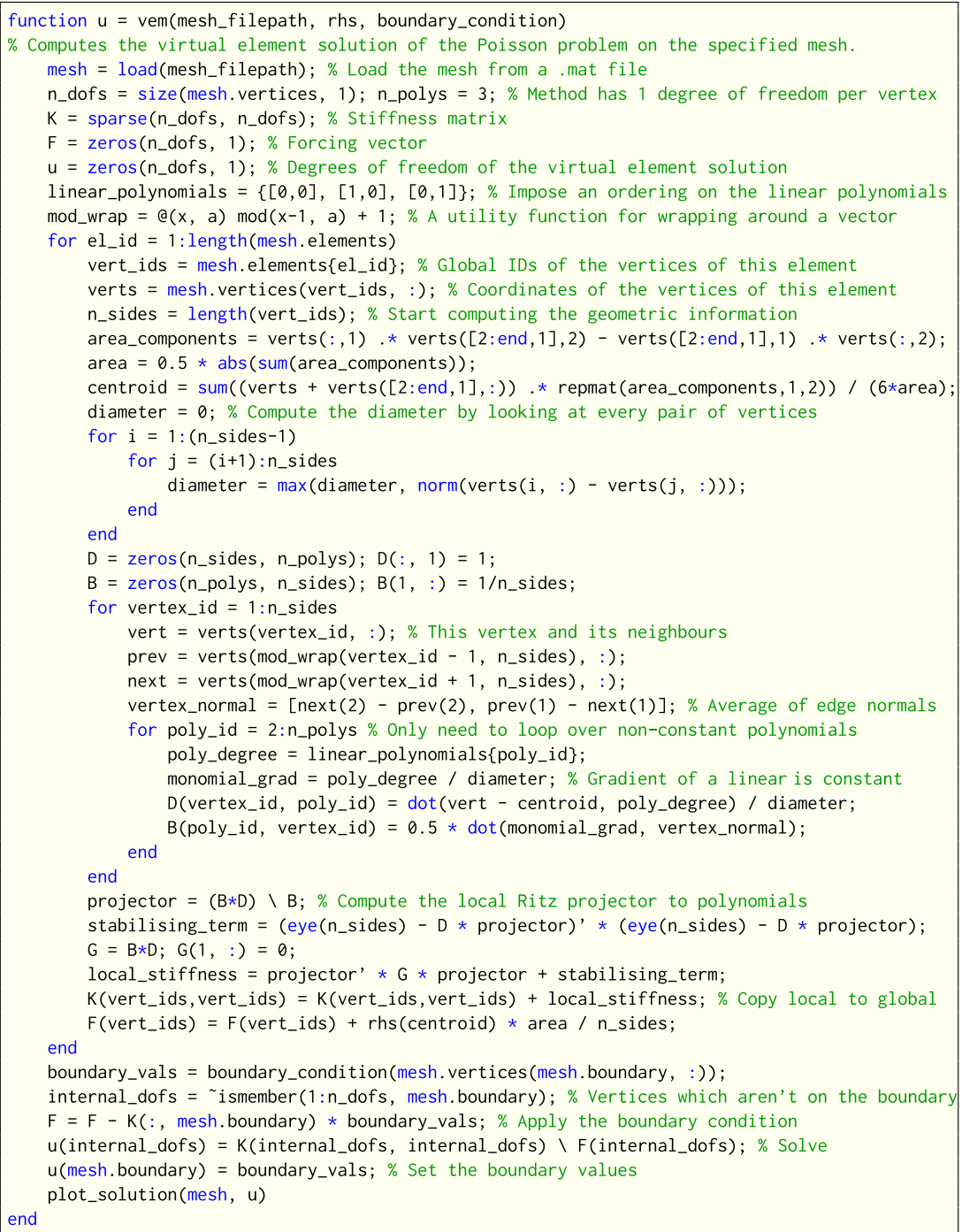

Listing 1 The file vem.m, implementing the virtual element method

and various sample mesh files, illustrated in Fig. 1. Examples of how to use the code are given in Section 5 .

\subsection{The polygonal mesh}

The mesh is loaded into a structure named mesh from a binary MATLAB . mat file (the path to which is specified by the input parameter mesh_filepath) containing a matrix named vertices which specifies the coordinates of a mesh vertex on each row, a cell array named elements containing vectors of indices indicating the 


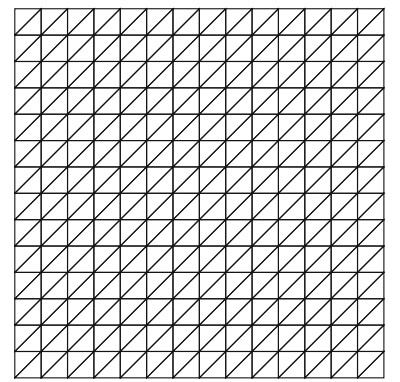

(A) Triangles

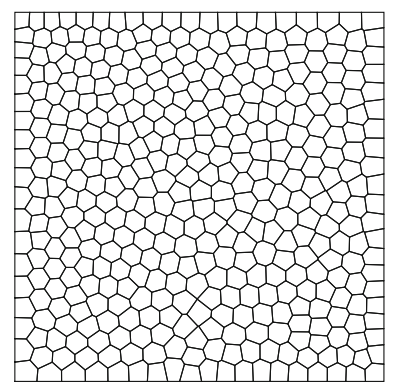

(D) Smoothed Voronoi

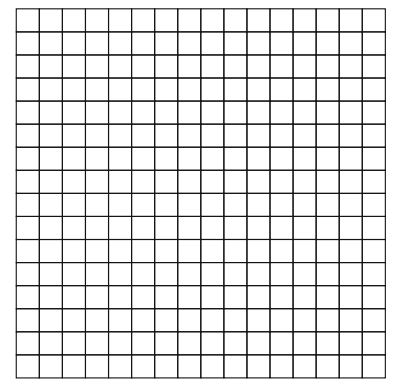

(B) Squares

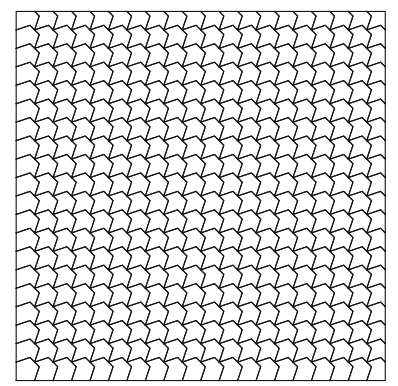

(E) Non-convex

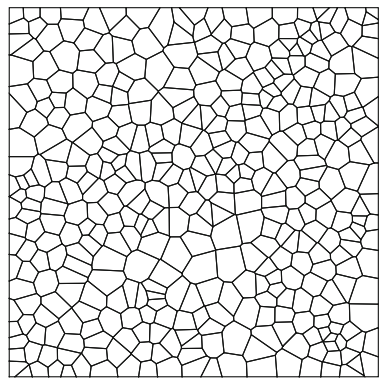

(C) Voronoi

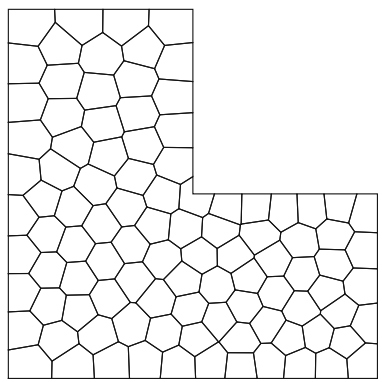

(F) L-shaped domain

Fig. 1 The sample meshes available along with the code

vertices which make up each element in an anti-clockwise order around the element, and a vector named boundary containing the indices of the vertices which lie on the boundary of the mesh. Illustrated in Fig. 1 are several examples of such meshes, available alongside the code from NETLIB. This information can also be generated in the same format using the Voronoi mesh generator PolyMesher [28], also written in MATLAB.

\subsection{Initialisation}

The input arguments to the code are mesh_filepath which contains the path to the mesh file, as described above, the function handle rhs which is a handle to a function implementing the forcing function of the PDE, and the function handle boundary_condition, which should be a handle to a function implementing the boundary condition of the PDE. Examples of such input arguments are given in Section 5.

The initialisation step of the code (lines 1-22) simply sets up various variables which will be useful to us later. In the interests of efficiency, we use a sparse matrix $\mathrm{K}$ to represent the stiffness matrix. In this step, we also define the cell array linear_polynomials containing three pairs of numbers indicating the degree 
of the associated polynomial in the $x$ and $y$ directions. Thus, the index of a specific polynomial in this array is taken to be the index of the polynomial in the basis $\mathcal{M}_{E}$. We note that the ordering imposed in the code coincides with the ordering in (4.1), although this choice is arbitrary.

Some extra element-specific initialisation also takes place within the loop over all mesh elements to compute various geometric properties of each element. The vector vert_ids contains the global indices of the vertices forming the element $E$ with an anti-clockwise ordering. As well as providing us with a means of looking up the coordinates of the vertices using the mesh structure, this also provides us with a very simple way to identify the global index of a particular local degree of freedom. This is possible because the indices of the degrees of freedom of the global virtual element space can be taken to be just the global indices of their associated vertices. Similarly, the local indices of the vertices of the element $E$ dictate the local index of the associated degree of freedom. Therefore, the $i$ th entry in the vector vert_ids provides the global index of the $i$ th local vertex and therefore also the global index of its associated local degree of freedom. Having access to this 'local to global' mapping is absolutely crucial when trying to assemble the global stiffness matrix and forcing vector from their local counterparts. When implementing more complex methods this sort of bookkeeping can quickly become very cumbersome, although here we are able to exploit the very simple arrangement of the degrees of freedom. Because of this property, we use the variable $\mathrm{n}_{-} \mathrm{s}$ ides to represent both the number of sides of $E$ and equivalently the number of local degrees of freedom of $V_{h}^{E}$.

The variable area denotes $|E|$ and is computed using the formula

$$
|E|=\frac{1}{2}\left|\sum_{i=1}^{N^{E}} x_{i} y_{i+1}-x_{i+1} y_{i}\right|,
$$

where $\left(x_{i}, y_{i}\right)$ are the coordinates of the vertex $v_{i}$, and the indexing is understood to wrap within the range 1 to $N^{E}$. In the code this is accomplished using the utility function mod_wrap, which modifies the standard mod function to produce output in the range 1 to $N^{E}$ rather than the range 0 to $N^{E}-1$. This modification to the mod function is necessary because arrays in MATLAB start at index 1, not 0 . The centroid $\left(x_{E}, y_{E}\right)$ of the element is stored in the vector centroid and calculated using the usual formula:

$$
\begin{aligned}
& x_{E}=\frac{1}{6|E|} \sum_{i=1}^{N^{E}}\left(x_{i}+x_{i+1}\right)\left(x_{i} y_{i+1}-x_{i+1} y_{i}\right), \\
& y_{E}=\frac{1}{6|E|} \sum_{i=1}^{N^{E}}\left(y_{i}+y_{i+1}\right)\left(x_{i} y_{i+1}-x_{i+1} y_{i}\right),
\end{aligned}
$$

where the indices are again to be understood to wrap within the range 1 to $N^{E}$. In the code, we are able to combine some of the calculations which are necessary to find the area and centroid by storing the terms of the sum (4.3) in the vector area_components. 


\subsection{The Ritz projection and local stiffness matrix}

We focus initially on computing $\Pi^{E} \varphi_{i}^{E}$ for a single basis function $\varphi_{i}^{E}$. Since $\Pi^{E} \varphi_{i}^{E} \in$ $\mathcal{P}^{E} \subset V_{h}^{E}$, we have two different possible expansions for the projection $\Pi^{E} \varphi_{i}^{E}$, either in the basis of $\mathcal{P}^{E}$ or in that of $V_{h}^{E}$ :

$$
\Pi^{E} \varphi_{i}^{E}=\sum_{\alpha=1}^{N_{\mathcal{P}}} a_{i, \alpha} m_{\alpha}=\sum_{j=1}^{N^{E}} s_{i, j} \varphi_{j}^{E}
$$

Recalling (3.2) and using the fact that $\nabla m_{\beta}$ is a constant vector for the linear polynomial $m_{\beta}$, we find that

$$
\begin{aligned}
\sum_{\alpha=1}^{N_{\mathcal{P}}} a_{i, \alpha}\left(\nabla m_{\alpha}, \nabla m_{\beta}\right)_{0, E} & =\sum_{j=1}^{N^{E}} \int_{e_{j}} v_{h} n_{e_{j}} \cdot \nabla m_{\beta} d_{s} \\
& =\sum_{j=1}^{N^{E}} \frac{\left|e_{j}\right|}{2}\left(\varphi_{i}^{E}\left(v_{j}\right)+\varphi_{i}^{E}\left(v_{j+1}\right)\right) n_{e_{j}} \cdot \nabla m_{\beta} \\
& =\frac{\left|e_{i}\right| n_{e_{i}}+\left|e_{i-1}\right| n_{e_{i-1}}}{2} \cdot \nabla m_{\beta},
\end{aligned}
$$

for any $m_{\beta} \in \mathcal{M}_{E}$, where in the last equality we have used the Lagrangian property of the basis functions $\varphi_{i}^{E}$ at the vertices of $E$ to determine that $\varphi_{i}^{E}$ is only non-zero on the two edges $e_{i}$ and $e_{i-1}$ which meet at the vertex $v_{i}$. As shown in [13], this can be further simplified to

$$
\sum_{\alpha=1}^{N_{\mathcal{P}}} a_{i, \alpha}\left(\nabla m_{\alpha}, \nabla m_{\beta}\right)_{0, E}=\frac{1}{2}\left|\widehat{e}_{i}\right| n_{\widehat{e}_{i}} \cdot \nabla m_{\beta},
$$

where we have denoted by $\widehat{e}_{i}$ the line segment connecting the vertices $v_{i-1}$ and $v_{i+1}$, and $n_{\widehat{e}_{i}}$ is the unit normal to $\widehat{e}_{i}$ such that $n_{\widehat{e}_{i}} \cdot n_{e_{j}} \geq 0$ for $j=i, i-1$ (Fig. 2).

Fig. 2 An illustration of the labelling of the various geometric attributes on each element. Vertices are labelled as $v_{i}$, the edge connecting $v_{i}$ to $v_{i+1}$ is denoted by $e_{i}$, and $\widehat{e}_{i}$ denotes the line segment connecting $v_{i-1}$ and $v_{i+1}$. The outward unit normal in each case is denoted by $n$ with the appropriate subscript

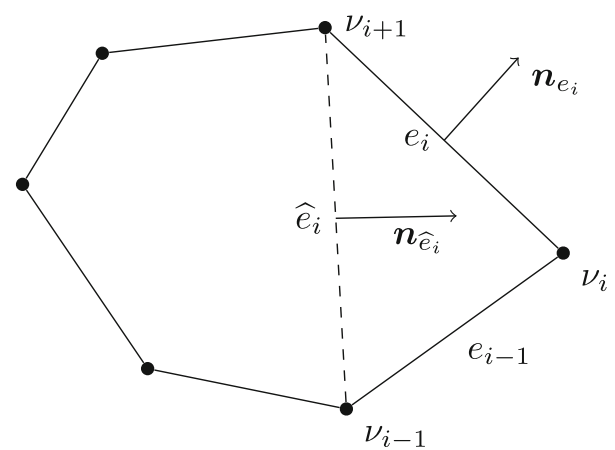


In view of this, and following [7], we introduce the matrix $G \in \Re^{N_{\mathcal{P}} \times N_{\mathcal{P}}}$ and the vector $B_{i} \in \mathfrak{R}^{N_{\mathcal{P}}}$ such that

$$
G_{\beta, \alpha}=\left(\nabla m_{\alpha}, \nabla m_{\beta}\right)_{0, E}, \quad \quad B_{\beta, i}=\frac{1}{2}\left|\widehat{e}_{i}\right| n_{\widehat{e}_{i}} \cdot \nabla m_{\beta},
$$

to encode the conditions above. The problem here is that the first row (and column) of $G$ and $B_{i}$ are zero, since the gradient of a constant function is zero, and therefore, $G$ is rank deficient. This is overcome by using the second condition in the definition (3.1) of $\Pi^{E}$, defining

$$
\widetilde{G}_{\beta, \alpha}=\left\{\begin{array}{ll}
\frac{1}{N^{E}} \sum_{j=1}^{N^{E}} m_{\alpha}\left(v_{j}\right) & \text { if } \beta=1, \\
G_{\beta, \alpha} & \text { otherwise, }
\end{array} \quad \widetilde{B}_{\beta, i}= \begin{cases}\frac{1}{N^{E}} & \text { if } \beta=1, \\
B_{\beta, i} & \text { otherwise }\end{cases}\right.
$$

Therefore, the coefficients $a_{i, \alpha}$ can be calculated by solving the (full rank) matrix equation

$$
\widetilde{G} a_{i}=\widetilde{B}_{i}
$$

Defining the matrix $\widetilde{B} \in \mathfrak{R}^{N_{\mathcal{P}} \times N^{E}}$ such that its $i$ th column is the vector $\widetilde{B}_{i} \in \mathfrak{R}^{N_{\mathcal{P}}}$, we obtain the matrix equation

$$
\widetilde{G} \Pi=\widetilde{B},
$$

where $\Pi \in \mathfrak{R}^{N_{\mathcal{P}} \times N^{E}}$ is the matrix representation of the Ritz projector $\Pi^{E}$, taking a vector of coefficients of a function expressed in terms of the basis of $V_{h}^{E}$ to a vector of coefficients of the basis of $\mathcal{P}_{E}$, and has $a_{i}$ as its $i$ th column.

We also introduce the matrix $D \in \mathfrak{R}^{N^{E} \times N_{\mathcal{P}}}$ with $D_{i, \alpha}:=\operatorname{dof}_{i}\left(m_{\alpha}\right)$ as a one-way 'change of basis' matrix for re-expressing polynomials in terms of the basis of $V_{h}^{E}$. It is easy to check from (3.2) that we may use $D$ as a shortcut to compute $G$ and $\widetilde{G}$, since

$$
G=B D, \quad \widetilde{G}=\widetilde{B} D,
$$

meaning we can compute the projection matrix as

$$
\Pi=(\widetilde{B} D)^{-1} \widetilde{B} .
$$

Since $\Pi$ transforms a vector of coefficients representing a function expressed in the basis of $V_{h}^{E}$ into a vector of coefficients of the basis of $\mathcal{P}_{E}$, the matrix $D \Pi$ then corresponds the same projector although with the resultant polynomial expressed in terms of the basis of $V_{h}^{E}$.

Finally, we can compute the local stiffness matrix as

$$
K^{E}=\Pi^{\top} G \Pi+(\mathrm{I}-D \Pi)^{\top}(\mathrm{I}-D \Pi),
$$

where $\mathrm{I} \in \mathfrak{R}^{N^{E} \times N^{E}}$ denotes the identity matrix. The first term of this sum corresponds to the consistency term of the discrete bilinear form, and the second term corresponds to the stabilising term.

The code which computes the matrix form of the local Ritz projector and the local stiffness matrix is given in lines $23-41$ of Listing 1 . The first task (lines 23-24 of Listing 1) is to initialise the two matrices $D$ and B representing their namesakes $D \in \mathfrak{R}^{N^{E} \times N_{\mathcal{P}}}$ and $B \in \mathfrak{R}^{N_{\mathcal{P}} \times N^{E}}$. For each of these matrices, we can immediately calculate the elements corresponding to the constant polynomial basis function $m_{1}$. In the case of $D$, every element of the first column contains the value 1 since the 
constant function is 1 everywhere, while it may be observed from (4.6) that every element in the first row of $B$ is equal to $N^{E}-1$.

However, the remaining elements of $D$ and $B$ must be computed separately for each of the basis polynomials with total degree equal to 1 , and for each local degree of freedom. Computing the entries of $D$ is a straightforward task, since it just involves evaluating the basis monomials at the vertices of the polygon.

For the entries of $B$, however, we must evaluate the second expression in (4.6). The quantity $\left|\widehat{e}_{i}\right| n_{\widehat{e}_{i}}$ is simple to calculate, since it is just the vector

$$
\left|\widehat{e}_{i}\right| n_{\widehat{e}_{i}}=\left(y_{i+1}-y_{i-1}, x_{i-1}-x_{i+1}\right)^{\top}
$$

due to the anti-clockwise orientation of the vertices around $E$. In the code, this result is stored in the variable vertex_normal, so named because it can be interpreted as a weighted normal vector at the vertex $v_{i}$. Again, the indices here are understood to wrap within the range 1 to $N^{E}$.

To compute the entries of $B$ we also need to evaluate $\nabla m_{\beta}$. Since $m_{\beta}$ is a linear polynomial, its gradient is simply a constant vector, and from the definition of $\mathcal{M}_{E}$ it is clear that

$$
\nabla m_{2}=\left(h_{E}^{-1}, 0\right)^{\top}, \quad \nabla m_{3}=\left(0, h_{E}^{-1}\right)^{\top},
$$

and hence by representing the polynomial degree of $m_{\beta}$ in the $x$ and $y$ directions using a vector with one entry 1 and one entry 0 , as in the cell array linear_polynomials, the gradient can be very simply calculated by just dividing by $h_{E}$.

With the matrices $D$ and $B$ computed, we are in a position to use (4.7) to calculate the matrix $\Pi$ representing the projector $\Pi^{E}$, as shown on line 37 of Listing 1, and stored in the variable projector. Consequently, with $D, B$ and $\Pi$ at our disposal, the local stiffness matrix can be computed as in (4.8).

The final step of this section is to add the elements of the local stiffness matrix to the positions in the global stiffness matrix corresponding to the associated global degrees of freedom. This is accomplished on line 41 through the local to global mapping discussed in Section 4.2.

\subsection{The local forcing vector}

To calculate the local forcing vector given in (4.2), we must first compute the projection $\Pi_{0}^{E} f$. By definition, this satisfies

$$
\int_{E} \Pi_{0}^{E} f \mathrm{~d} x=\int_{E} f \mathrm{~d} x,
$$

which, because we are projecting to constants, can be simplified to

$$
\Pi_{0}^{E} f=\frac{1}{|E|} \int_{E} f \mathrm{~d} x \approx f\left(x_{E}, y_{E}\right),
$$

where in the last relation we have used the barycentric quadrature rule on the polygon to approximate the integral. Since we are only considering the linear virtual element method, this is sufficiently accurate to ensure the optimal order of convergence in 
the $H^{1}(\Omega)$ norm. It is the use of this quadrature which produces the requirement in Section 3 that the element must contain its own centroid. Clearly, more general integration methods are possible (for example by triangulating the element or using more advanced techniques such as $[19,20,24,26])$, although for the sake of simplicity this is not something we pursue here. Since each basis function of $V_{h}^{E}$ is defined to be 1 at a single vertex and 0 at the others, we can express

$$
F_{j}^{E}=\int_{E} \overline{\varphi_{j}^{E}} \Pi_{0}^{E} f \mathrm{~d} x \approx \int_{E} \frac{f\left(x_{E}, y_{E}\right)}{N^{E}} \mathrm{~d} x=\frac{|E|}{N^{E}} f\left(x_{E}, y_{E}\right) .
$$

The code to compute this and store the result in the appropriate positions in the global forcing vector is on line 42 of Listing 1.

\subsection{Applying the boundary conditions}

The final step involves condensing the degrees of freedom associated with the boundary of the domain out of the linear system using the boundary condition, solving the resulting matrix equation, and re-applying the boundary data to the computed solution. This part of the procedure is exactly the same as for a standard finite element method, but for completeness we briefly review the process here.

Using the subscript $B$ to denote the indices of degrees of freedom on $\partial \Omega$ and $I$ to denote those in the interior of $\Omega$, the matrix problem $K U=F$ can be expressed as

$$
\left[\begin{array}{ll}
K_{I I} & K_{I B} \\
K_{B I} & K_{B B}
\end{array}\right]\left[\begin{array}{c}
U_{I} \\
U_{B}
\end{array}\right]=\left[\begin{array}{l}
F_{I} \\
F_{B}
\end{array}\right]
$$

where $K_{I B}=K_{B I}^{\top}$ by the symmetry of the bilinear form. Therefore, since $U_{B}$ is known, we find $U_{I}$ by solving the problem

$$
K_{I I} U_{I}=F_{I}-K_{I B} U_{B} .
$$

This is realised on lines $46-47$ of the code, where we only store the result of solving the matrix system to the positions of the vector $u$ which correspond to internal degrees of freedom while the values of the boundary degrees of freedom are set separately on line 48 .

The final line of the code uses the auxiliary function plot_solution, also provided in the NETLIB package, to plot the vertex values of the virtual element solution and the mesh using MATLAB's patch function.

Remark 1 (More general boundary conditions) Problems involving more general boundary conditions can also be implemented in a similar manner as for the finite element method. Suppose that the domain boundary is split into two components $\Gamma_{D}$ and $\Gamma_{N}$ such that $\Gamma_{D} \cup \Gamma_{N}=\partial \Omega$ and $\Gamma_{D} \cap \Gamma_{N}=\emptyset$. Consider a problem posed with mixed type boundary conditions such that, for sufficiently regular Neumann boundary data $g_{N}$,

$$
\begin{array}{cl}
-\Delta u=f & \text { in } \Omega, \\
u=0 & \text { on } \Gamma_{D}, \\
n \cdot \nabla u=g_{N} & \text { on } \Gamma_{N} .
\end{array}
$$


The case of mixed boundary conditions involving non-homogeneous Dirichlet data can also be handled as shown above. The weak form of this problem then reads: find $u \in H_{\Gamma_{D}}^{1}(\Omega)$ such that

$$
(\nabla u, \nabla v)=(f, v)-\int_{\Gamma_{N}} g_{N} v d s \quad \forall v \in H_{\Gamma_{D}}^{1}(\Omega),
$$

where $H_{\Gamma_{D}}^{1}(\Omega)$ denotes the subspace of $H^{1}(\Omega)$ consisting of functions with vanishing trace on $\Gamma_{D}$. A virtual element method for this problem can then be written as: find $u_{h} \in V_{h}$ such that

$$
a_{h}\left(u_{h}, v_{h}\right)=\ell_{h}\left(v_{h}\right)-\int_{\Gamma_{N}} g_{N} v_{h} d s \quad \forall v_{h} \in V_{h},
$$

where $a_{h}$ and $\ell_{h}$ are as before and $V_{h}$ denotes a virtual element space incorporating the homogeneous Dirichlet boundary data on $\Gamma_{D}$. The left and right-hand sides of this problem can still be evaluated exactly since $v_{h}$ is a known linear polynomial on each edge of the mesh and $g_{N}$ is a known function on $\Gamma_{N}$. Consequently, only the extra vector corresponding to the term on the Neumann part of the boundary would need to be computed in the code to implement mixed boundary conditions.

Remark 2 (Extension to three spatial dimensions) Implementing the virtual element method for problems in three spatial dimensions on polyhedral meshes requires the use of the so-called enhanced virtual element spaces introduced in [1]. In these modified local function spaces, it becomes possible to compute the local $L^{2}$-orthogonal projection of virtual element functions onto the polynomial subspace on each mesh element and face. The matrix formulation of this method and the algorithm for computing the $L^{2}$-orthogonal projection operator is discussed in [7]. These projection operators are required to evaluate the right hand side of (3.2) when computing the local Ritz projection. This is because a virtual element function in three spatial dimensions is not a polynomial on each (in general polygonal) face of the element, but rather belongs to a virtual element space defined on that planar face.

\section{Sample usage}

The full code is available as the na45 package in the NUMERALGO library from Netlib. The software is distributed as a compressed archive file, and can be installed be decompressing the file to produce the directory "vem_50lines", which contains the code. More information is provided in the README.txt file, contained in the package.

As described in Section 4, two different implementations of the right hand side forcing function and boundary condition are provided with the code. The first of these is designed for use on a square-shaped domain, while the second is designed for use on an L-shaped domain. The sample solution on the square-shaped domain is shown in Fig. 3a on the Voronoi mesh of Fig. 1c. This figure can be produced by navigating to the directory containing the file vem.m within MATLAB and executing 


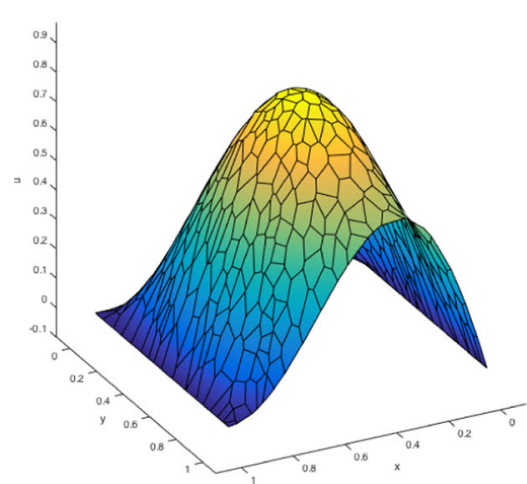

(A) The sample solution on a square domain, using the Voronoi mesh in Figure 1(c).

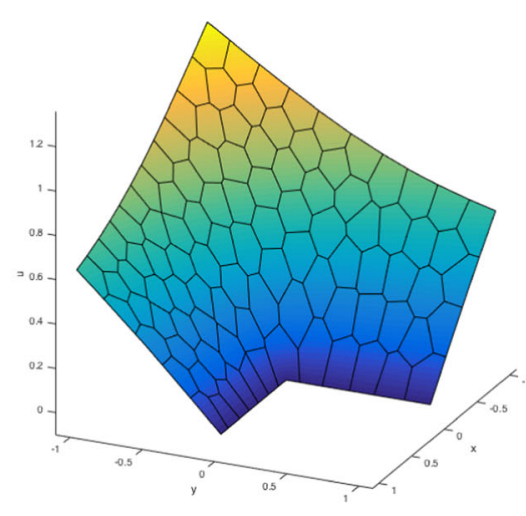

(B) The sample solution on an L-shaped domain, using the mesh in Figure 1(f).

Fig. 3 The virtual element approximations to the solutions of the two sample problems supplied with the code

vem('meshes/voronoi.mat', @square_domain_rhs, @square_domain_boundary_condition);

to provide the code with an appropriate mesh, forcing function, and boundary condition. The other sample meshes of the square domain could equivalently be used here by simply changing the first argument in the function call to be the path to the desired mesh. For instance, replacing the first argument with ' meshes/triangles.mat', ' meshes/squares. mat', 'meshes/smoothed-voronoi.mat', or 'meshes/non-convex.mat' would use the meshes shown in Fig. 1a, b, d, e, respectively.

Similarly, the sample solution for the L-shaped domain is shown in Fig. 3a. This plot is produced by calling the vem function with the parameters:

$$
\begin{aligned}
& \text { vem ('meshes/L-domain.mat', @L_domain_rhs, } \\
& \text { @L_domain_boundary_condition); }
\end{aligned}
$$

which corresponds to the standard problem on an L-shaped domain.

\section{Conclusions and extensions}

We have presented a 50-line MATLAB implementation of the linear virtual element method introduced in [6] for solving the Poisson problem on polygonal meshes in two spatial dimensions. The code for this is provided with this paper alongside several example polygonal meshes. To the best of our knowledge, this is the first publicly available implementation of the virtual element method. It is clear from the literature surrounding the method that its capabilities extend far beyond what is presented here, although the intention behind this work is to exemplify how the method can be implemented in practice, in the simplest possible setting. The ideas we present here 
can, however, be generalised to much more complicated situations by applying similar processes to compute the various required terms. The possible extensions of this code are endless: the implementation of higher order methods, more general elliptic operators including lower order terms and non-constant coefficients [8, 14], basis functions with higher global regularity properties [10], mesh adaptation driven by a posteriori error indicators [12], or the consideration of time dependent problems [30] to name but a few.

Acknowledgments The support of the EPSRC whilst producing this work is gratefully acknowledged.

Open Access This article is distributed under the terms of the Creative Commons Attribution 4.0 International License (http://creativecommons.org/licenses/by/4.0/), which permits unrestricted use, distribution, and reproduction in any medium, provided you give appropriate credit to the original author(s) and the source, provide a link to the Creative Commons license, and indicate if changes were made.

\section{References}

1. Ahmad, B., Alsaedi, A., Brezzi, F., Marini, L.D., Russo, A.: Equivalent projectors for virtual element methods. Computers \& Mathematics with Applications 66(3), 376-391 (2013)

2. Alberty, J., Carstensen, C., Funken, S.A.: Remarks around 50 lines of Matlab: short finite element implementation. Numer Algorithms 20(2-3), 117-137 (1999)

3. Antonietti, P.F., Beirão da Veiga, L., Mora, D., Verani, M.: A stream virtual element formulation of the Stokes problem on polygonal meshes. SIAM J. Numer. Anal. 52(1), 386-404 (2014)

4. Ayuso de Dios, B., Lipnikov, K., Manzini, G.: The nonconforming virtual element method. ESAIM: M2AN 50(3), 879-904 (2016)

5. Bahriawati, C., Carstensen, C.: Three MATLAB implementations of the lowest-order RaviartThomas MFEM with a posteriori error control. Comput. Methods Appl. Math. 5(4), 333-361 (2005). (electronic)

6. Beirão da Veiga, L., Brezzi, F., Cangiani, A., Manzini, G., Marini, L.D., Russo, A.: Basic principles of virtual element methods. Math. Models Methods Appl. Sci. 23(1), 199-214 (2013)

7. Beirão da Veiga, L., Brezzi, F., Marini, L.D., Russo, A.: The hitchhiker's guide to the virtual element method. Math. Models Methods Appl. Sci. 24(8), 1541-1573 (2014)

8. Beirão da Veiga, L., Brezzi, F., Marini, L.D., Russo, A.: Virtual element method for general secondorder elliptic problems on polygonal meshes. Math. Models Methods Appl. Sci. 26(4), 729-750 (2016)

9. Beirão da Veiga, L., Lovadina, C., Russo, A.: Stability Analysis for the Virtual Element Method. arXiv (2016)

10. Beirão da Veiga, L., Manzini, G.: A virtual element method with arbitrary regularity. IMA J. Numer. Anal. 34(2), 759-781 (2014)

11. Brezzi, F., Falk, R.S., Marini, L.D.: Basic principles of mixed virtual element methods. ESIAM: M2AN 48(4), 1227-1240 (2014)

12. Cangiani, A., Georgoulis, E.H., Pryer, T., Sutton, O.J.: A posteriori error estimates for the virtual element method. arXiv (2016)

13. Cangiani, A., Manzini, G., Russo, A., Sukumar, N.: Hourglass stabilization and the virtual element method. Int. J. Numer. Meth. Engng. 102(3-4), 404-436 (2015)

14. Cangiani, A., Manzini, G., Sutton, O.J.: Conforming and nonconforming virtual element methods for elliptic problems. IMA J. Numer. Anal. (2016)

15. Gain, A.L., Talischi, C., Paulino, G.H.: On the virtual element method for three-dimensional linear elasticity problems on arbitrary polyhedral meshes. Comput. Methods Appl. Mech. Engrg. 282, 132160 (2014)

16. Hecht, F.: New development in FreeFem++. J. Numer. Math. 20, 3-4, 251-265 (2012) 
17. Logg, A., Mardal, K.-A., Wells, G.N., et al.: Automated solution of differential equations by the finite element method. Springer (2012)

18. Manzini, G., Russo, A., Sukumar, N.: New perspectives on polygonal and polyhedral finite element methods. Math. Models Methods Appl. Sci. 24(8), 1665-1699 (2014)

19. Mousavi, S.E., Sukumar, N.: Numerical integration of polynomials and discontinuous functions on irregular convex polygons and polyhedrons. Comput. Mech. 47(5), 535-554 (2011)

20. Mousavi, S.E., Xiao, H., Sukumar, N.: Generalized Gaussian quadrature rules on arbitrary polygons. Int. J. Numer. Meth. Engng. 82(1), 99-113 (2010)

21. Perugia, I., Pietra, P., Russo, A.: A Plane Wave Virtual Element Method for the Helmholtz Problem. ESIAM: M2AN 50(3), 783-808 (2016)

22. Rjasanow, S., Weißer, S.: Higher order BEM-based FEM on polygonal meshes. SIAM J. Numer. Anal. 50(5), 2357-2378 (2012)

23. Sigmund, O.A.: 99 line topology optimization code written in Matlab. Struct. Multidiscip. Optim. 21(2), 120-127 (2001)

24. Sommariva, A., Vianello, M.: Product Gauss cubature over polygons based on Green's integration formula. BIT Numer. Math. 47(2), 441-453 (2007)

25. Strouboulis, T., Babuška, I., Copps, K.: The design and analysis of the generalized finite element method. Comput. Methods Appl. Mech. Engrg. 181(1-3), 43-69 (2000)

26. Sudhakar, Y., Moitinho de Almeida, J.P., Wall, W.A.: An accurate, robust, and easy-to-implement method for integration over arbitrary polyhedra: application to embedded interface methods. J. Comput. Phys 273, 393-415 (2014)

27. Sukumar, N., Tabarraei, A.: Conforming polygonal finite elements. Int. J. Numer. Meth. Engng. 61(12), 2045-2066 (2004)

28. Talischi, C., Paulino, G.H., Pereira, A., Menezes, I.F.M.: PolyMesher: a general-purpose mesh generator for polygonal elements written in Matlab. Struct. Multidiscip. Optim. 45(3), 309-328 (2012)

29. Taylor, R.: FEAP - a finite element analysis program. University of California at Berkeley (2013). Version 8.4

30. Vacca, G., Beirão da Veiga, L.: Virtual element methods for parabolic problems on polygonal meshes. Numer. Methods Partial Differential Equations 31(6), 2110-2134 (2015) 